LOGES, V.; LEMOS, M.A.; RESENDE, L.V.; MENEZES, D.; CANDEIA, J.A.; SANTOS, V.F. Correlações entre caracteres agronômicos associados à resistência a tripes em cebola. Horticultura Brasileira, Brasília, v.22, n.3, p.624-627, jul-set 2004.

\title{
Correlações entre caracteres agronômicos associados à resistência a tripes em cebola
}

\author{
Vivian Loges ${ }^{1}$; Margarida Agostinho Lemos ${ }^{1}$; Luciane Vilela Resende ${ }^{1}$; Dimas Menezes ${ }^{1}$; Jonas Araújo \\ Candeia $^{2}$; Venézio Felipe dos Santos ${ }^{2}$ \\ ${ }^{1}$ UFRPE, Depto. fitotecnia, Av. D. Manoel de Medeiros, s/n, 52171-900 Recife-PE; E-mail: vloges@ yahoo.com; ${ }^{2}$ IPA, Av. Gal. San \\ Martin, 1371, 50761-000 Recife-PE: E-mail: ipa@ipa.br
}

\section{RESUMO}

Foram determinadas as correlações genotípicas, fenotípicas e ambientais entre caracteres agronômicos e resistência a Thrips tabaci em 62 progênies de meio-irmãos da cultivar ValeOuro IPA-11 de cebola. O experimento foi conduzido em campo, em Juazeiro (BA), com avaliações aos 48; 69 e 93 dias após o transplantio (DAT). A produção de bulbos comerciais (PBC) apresentou correlações genotípicas com comprimento de folhas $(\mathrm{CF})(0,63 * *)$; número total de folhas (NTF) $(0,74 * *)$; número de folhas danificadas (NFD) $\left(0,74^{* *}\right)$; peso médio de bulbos comerciais (PMBC) $\left(0,54^{* *}\right)$; porcentagem de bulbos comerciais $(\% \mathrm{BC})(0,61 * *)$; e porcentagem de bulbos não comerciais (\%BNC) $\left(-0,63^{* *}\right)$. Foram observadas correlações genotípicas do número de ninfas de $T$. tabaci entre as duas folhas centrais completamente desenvolvidas (NINFA), aos 69 DAT com ângulo entre as folhas centrais (ANG) $\left(-0,76^{* *}\right)$; NTF $\left(0,46^{* *}\right)$; $\% \mathrm{BC}\left(-0,35^{* *}\right)$; e porcentagem de plantas improdutivas $(\% \mathrm{I})$ $(0,39 * *)$. Estas correlações indicam que menos ninfas ocorre em plantas com menor NTF e maior ANG. Por sua vez, plantas com menor NTF apresentam maior ANG $(-0,92 * *)$. Porém, ressalta-se que o NTF correlacionou-se positivamente com PBC, PMBC $\left(0,45^{* *}\right)$ e $\% \mathrm{BC}\left(0,43^{* *}\right)$. As correlações genotípicas positivas de NFD com PBC, PMBC $(0,40 * *)$ e $\% B C(0,51 * *)$, sugerem que esta população apresenta resistência por tolerância.

Palavras-chave: Allium cepa, Thrips tabaci, melhoramento, resistência de plantas a insetos, arquitetura da planta, produção.

\begin{abstract}
Correlation of agronomic characters associated to thrips resistance in onion

Genotypic, phenotypic and environmental correlation between agronomic characteristics and Thrips tabaci resistance were determined in 62 half-sibs progenies of onion ValeOuro IPA-11 cultivar. The experiment was conducted under field conditions in Juazeiro, Bahia State, Brazil, with evaluations at 48; 69 and 93 days after transplant (DAT). Commercial bulb yield (PBC) presented genotypic correlation with leaf length $(\mathrm{CF})\left(0,63^{* *}\right)$; leaf number $(\mathrm{NTF})\left(0,74^{* *}\right)$; damaged leaf number (NFD) $\left(0,74^{* *}\right)$; commercial bulb average weight (PMBC) $\left(0,54^{* *}\right)$; commercial bulb yield $(\% \mathrm{BC})(0,61 * *)$; and non-commercial bulb yield $(\% \mathrm{BNC})\left(-0,63^{* *}\right)$. Genotypic correlations were observed in the number of ninfas of T. tabaci between two fully developed central leaves (NINFA), at 69 DAT and central leaf angle (ANG) $\left(-0,76^{* *}\right)$; $\operatorname{NTF}\left(0,46^{* *}\right) ; \% \mathrm{BC}\left(-0,35^{* *}\right)$; and yield of unproductive plants $(\% \mathrm{I})$ $(0,39 * *)$. These correlations indicate that lower NINFA numbers occur in plants with lower NTF and higher ANG. Lower NTF plants, on the other hand, have higher ANG (-0,92**). Never thelessNTF correlated positively with $\mathrm{PBC}, \mathrm{PMBC}\left(0,45^{* *}\right)$ and $\% \mathrm{BC}\left(0,43^{* *}\right)$. Positive genotypic correlations between NFD and PBC, PMBC $\left(0,40^{* *}\right)$ and $\% \mathrm{BC}(0,51 * *)$, imply that this population presents resistance through tolerance.
\end{abstract}

Keywords: Allium cepa, Thrips tabaci, breeding, insect plant resistance, plant architecture, yield.

\section{(Recebido para publicação em 16 de julho de 2003 e aceito em 29 de abril de 2004)}

$\mathrm{P}$ rogramas de melhoramento de cebola devem basear-se em caracteres correlacionados com a produção, que apresentem alta herdabilidade (Candeia et al., 1986), e com resistência às pragas e doenças (Brewster, 1994). Entre as pragas dessa cultura, o Thrips tabaci é considerada a principal, podendo causar prejuízos consideráveis (Boiça Junior e Domingues, 1987; Sato, 1989). Apesar da importância desta praga, os caracteres correlacionados com a sua resistência ainda não foram bem esclarecidos.

A resistência de cebola ao tripes pode ser determinada por características da arquitetura da planta que mantêm baixa a infestação do inseto e por características fisiológicas que permitem à planta suportar os danos (Jones et al., 1935; Sato, 1989). Correlações negativas foram observadas entre a infestação de T. tabaci e caracteres como comprimento, peso, área total e número de folhas (Cartwright et al., 1995) e caracteres de produção como porcentagem de plantas sobreviventes, bulbos comerciais, produtividade e peso médio dos bulbos (Boiça Junior e Domingues, 1987).

A cultivar ValeOuro IPA-11 apresenta folhagem com mediana resistência aos danos por tripes, porém, o tipo e causas da resistência a esta praga não foram bem avaliados (Candeia et al., 1998). Este trabalho objetivou estimar as correlações genotípicas, fenotípicas e ambientais entre onze caracteres agronômicos em progênies de meio-irmãos de cebola, cultivar ValeOuro IPA-11, a fim de auxiliar no melhoramento quanto à produção e resistência a T. tabaci.

\section{MATERIAL E MÉTODOS}

O experimento foi conduzido em Juazeiro, na região do Submédio São Francisco, em blocos ao acaso, com três repetições, de julho a dezembro de 1998. Foram avaliadas 62 progênies de meioirmãos de cebola da cultivar ValeOuro IPA-11, obtidas a partir da colheita de sementes de plantas individuais, tomadas ao acaso, em um campo de produção de sementes na EE do IPA em Serra Talhada (PE). Esta cultivar desenvolvida pelo IPA (Candeia et al., 1997), oriunda do cruzamento entre a cv. Roxa IPA- 
Tabela 1. Estimativas dos coeficientes de correlação genotípica $\left(r_{G}\right)$, correlação fenotípica $\left(r_{F}\right)$ e ambiental $\left(r_{a}\right)$ entre caracteres de folha, avaliados aos 48 e 69 dias após o transplantio, em 62 progênies de meio-irmãos de cebola ValeOuro IPA-11. Juazeiro (BA), UFRPE, 1998.

\begin{tabular}{|c|c|c|c|c|c|c|c|c|c|}
\hline \multirow{2}{*}{ Caracter } & \multirow{2}{*}{ Correlação } & \multicolumn{2}{|c|}{ ANG ${ }^{1}$} & \multicolumn{2}{|c|}{ NTF } & \multicolumn{2}{|c|}{ NFD } & \multicolumn{2}{|c|}{ NINFA } \\
\hline & & 48 & 69 & 48 & 69 & 48 & 69 & 48 & 69 \\
\hline \multirow[t]{3}{*}{$\overline{\mathrm{CF}}$} & $\mathrm{rG}$ & $0,49^{* *}$ & $-0,57^{* *}$ & $0,97^{* *}$ & $0,19 \mathrm{~ns}$ & $0,09 \mathrm{~ns}$ & $0,31^{* *}$ & $0,78^{* *}$ & $-0,08 n s$ \\
\hline & $\mathrm{rF}$ & $-0,18 \mathrm{~ns}$ & $-0,16 n s$ & $0,49^{* *}$ & $0,42^{* *}$ & $0,25^{* *}$ & $0,45^{* *}$ & $0,13 \mathrm{~ns}$ & $0,29 * *$ \\
\hline & ra & $-0,38^{* *}$ & $0,16 \mathrm{~ns}$ & $0,41^{* *}$ & $0,49^{* *}$ & $0,09 \mathrm{~ns}$ & $0,50^{* *}$ & $0,03 \mathrm{~ns}$ & $0,35^{* *}$ \\
\hline \multirow[t]{3}{*}{$\overline{\mathrm{ANG}}$} & rG & & & $-0,30^{* *}$ & $-0,92^{* *}$ & $-0,81^{* *}$ & $-0,12 n s$ & $-0,55^{* *}$ & $-0,76^{* *}$ \\
\hline & $\mathrm{rF}$ & & & $-0,23 n s$ & $-0,37^{* *}$ & $-0,33^{* *}$ & $-0,37^{* *}$ & $-0,27^{* *}$ & $-0,39^{* *}$ \\
\hline & ra & & & $-0,22 \mathrm{~ns}$ & $-0,13 \mathrm{~ns}$ & $-0,23^{* *}$ & $0,00 \mathrm{~ns}$ & $-0,22 n s$ & $-0,27^{\text {* * }}$ \\
\hline \multirow[t]{3}{*}{$\overline{\text { NTF }}$} & rG & & & & & $0,38^{* *}$ & $0,07 \mathrm{~ns}$ & $0,29^{* *}$ & $0,46^{* *}$ \\
\hline & $\mathrm{rF}$ & & & & & $0,32^{* *}$ & 0,89 ** & $0,31^{* *}$ & $0,52^{* *}$ \\
\hline & $\mathrm{ra}$ & & & & & $0,31^{* *}$ & $0,82^{* *}$ & $0,35^{* *}$ & $0,55^{* *}$ \\
\hline \multirow[t]{3}{*}{$\overline{N F D}$} & $r G$ & & & & & & & $0,73^{* *}$ & $0,09 \mathrm{~ns}$ \\
\hline & $\mathrm{rF}$ & & & & & & & $0,72^{* *}$ & $0,44^{* *}$ \\
\hline & ra & & & & & & & $0,71^{* *}$ & $0,58^{* *}$ \\
\hline
\end{tabular}

$* *=\mathrm{P}<0,01$ e ns = não significativo pelo Teste $\mathrm{t}$.

${ }^{1} \mathrm{CF}$ - comprimento de folhas; ANG - ângulo entre as duas folhas centrais completamente desenvolvidas; NTF - número total de folhas; NFD - número de folhas danificadas por tripes; NINFA - número de ninfas nas duas folhas centrais completamente desenvolvidas.

3 e Belém IPA-9, foi melhorada para a região semi-árida do Nordeste, após oito ciclos de seleção massal. Apresenta folhas vigorosas, moderadamente eretas, de coloração verde escuro, muito cerosas e com alta produtividade.

O transplantio foi realizado 37 dias após a semeadura, no espaçamento de $0,20 \times 0,15 \mathrm{~m}$ e $1 \mathrm{~m}$ entre sulcos de irrigação, sem a seleção para tamanho de plantas. Os demais tratos culturais foram os adotados na região, porém sem o uso de agrotóxicos após o transplantio. Foram realizadas três avaliações: aos 48 dias após o transplantio (DAT), aos 69 DAT e na colheita aos 93 DAT.

Aos 48 e 69 DAT, cinco plantas forma coletadas ao acaso em parcelas com 2,50 m de comprimento, para avaliação dos caracteres de folhas: 1) comprimento de folhas (CF) da bainha até o ápice da maior folha; 2) ângulo entre as duas folhas centrais completamente desenvolvidas (ANG), tomado com o auxílio de um transferidor a partir do eixo central da planta; 3 ) número total de folhas (NTF); 4) número de folhas danificadas por tripes (NFD); 5) número de ninfas nas duas folhas centrais completamente desenvolvidas (NINFA), segundo metodologia de Loges et al. (2000).

Aos 93 DAT, quando 50\% das plantas apresentaram tombamento da folhagem, todas as plantas da parcela $(8,5 \mathrm{~m}$ de comprimento) foram acondicionadas em sacos telados e levadas para local sombreado e bem arejado. Após dois dias de cura foram eliminadas as raízes e folhas para avaliação dos caracteres associados à produção:1) produção de bulbos não comerciais (PBNC) em kg/parcela (bulbos com diâmetro inferior a 40 $\mathrm{mm})$; 2) produção de bulbos comerciais (PBC) em kg/parcela (bulbos com diâmetro maior ou igual a $40 \mathrm{~mm}$ ); 3) peso médio de bulbos comerciais (PMBC) em $\mathrm{kg}$ (peso total de bulbos comerciais dividido pelo número de bulbos comerciais); 4) porcentagem de plantas improdutivas (\%) (plantas sem bulbificação em relação ao número de plantas sobreviventes); 5) porcentagem de bulbos não comerciais (\%BNC) (bulbos não comerciais em relação ao número de plantas sobreviventes); 6) porcentagem de bulbos comerciais (\%BC) (bulbos comerciais em relação ao número de plantas sobreviventes).

As análises estatísticas, foram efetuadas segundo o modelo matemático de Vencovsky e Barriga (1992), utilizando o programa GENES (Cruz, 1997) para o cálculo das covariâncias e os coeficientes de correlação genética, fenotípica e ambiental entre os cinco caracteres de folha avaliados aos 48 e 69 DAT, entre os seis caracteres associados à produção e entre todos eles. O nível de significância foi calculado pelo Teste t.

\section{RESULTADOS E DISCUSSÃO}

As correlações genotípicas foram superiores às fenotípicas, apresentaram o mesmo sinal e foram superiores às correlações ambientais em $60 \%$ das correlações. Estes valores indicam que as causas de variação genética e ambiental afetaram os caracteres através de mecanismos fisiológicos semelhantes (Falconer, 1981) e que há uma tendência de maior contribuição dos fatores genéticos do que ambientais nas correlações entre estes caracteres.

Amostras dos tripes enviadas para identificação indicaram a ocorrência apenas da espécie T. tabaci L. (Monteiro, 2001). Infestações maiores deste tripes foram associadas às plantas com maior número de folhas e que apresentam menores ângulos entre as folhas centrais. Isto pode ser observado nas correlações genotípicas, fenotípicas e ambientais, nas duas avaliações, as quais se mostraram positivas para NINFA e NTF, negativas para NINFA e ANG e para ANG e NTF (Tabela 1). Resultados semelhantes foram observados com a cultivar de cebola branca Nebuka (Coudriet et al., 1979) e em plantas de alho (Bortoli et al., 1995), com folhas de crescimento do tipo aberto e seção circular, que apresentaram menor número de ninfas e menor dano. A razão disto pode ser que plantas com folhas achatadas e com ponto de crescimento compacto por apresentarem menor abertura entre as folhas, promovem melhor abrigo para os tripes, protegendo-os contra inimigos naturais, ação dos inseticidas e minimizando as condições adversas do ambiente (Jones et al., 1935; Sato, 1989). Já Kendall e Capinera (1987) correlacionaram a produção de folhas 
Tabela 2. Estimativas dos coeficientes de correlação genotípica $\left(\mathrm{r}_{\mathrm{G}}\right)$, correlação fenotípica $\left(\mathrm{r}_{\mathrm{F}}\right)$ e ambiental $\left(\mathrm{r}_{\mathrm{a}}\right)$ para caracteres associados à produção em 62 progênies de meio-irmãos de cebola ValeOuro IPA-11. Juazeiro (BA), UFRPE, 1998.

\begin{tabular}{|c|c|c|c|c|c|c|}
\hline Caracter & Correlação & $\mathrm{PBC}^{1}$ & РMBC & $\%$ I & $\%$ BNC & $\% B C$ \\
\hline \multirow[t]{3}{*}{$\overline{P B N C}$} & $\mathrm{rG}$ & $-0,14 \mathrm{~ns}$ & $-0,81$ ** & $0,27^{* *}$ & $0,80^{* *}$ & $-0,77^{* *}$ \\
\hline & $\mathrm{rF}$ & $-0,44$ ** & $-0,40 * *$ & 0,36 ** & 0,85 ** & $-0,81$ ** \\
\hline & ra & $-0,61^{* *}$ & $-0,24^{* *}$ & $0,41^{\text {** }}$ & $0,87^{* *}$ & $-0,83^{* *}$ \\
\hline \multirow[t]{3}{*}{ PBC } & $r G$ & & 0,54 ** & $-0,18 \mathrm{~ns}$ & $-0,63$ ** & 0,61 ** \\
\hline & $\mathrm{rF}$ & & 0,50 ** & $-0,47$ ** & $-0,72$ ** & 0,74 * * \\
\hline & $\mathrm{ra}$ & & $0,50^{* *}$ & $-0,62^{* *}$ & $-0,77^{\star *}$ & $0,80^{* *}$ \\
\hline \multirow[t]{3}{*}{ PMBC } & $r G$ & & & $-0,14 \mathrm{~ns}$ & $-0,73$ ** & 0,70 * * \\
\hline & $\mathrm{rF}$ & & & $-0,15 \mathrm{~ns}$ & $-0,32$ ** & 0,31 * * \\
\hline & $\mathrm{ra}$ & & & $-0,16 n s$ & $-0,17 \mathrm{~ns}$ & $0,18 \mathrm{~ns}$ \\
\hline \multirow[t]{3}{*}{$\%$} & $\mathrm{rG}$ & & & & $0,38^{* *}$ & $-0,59^{* *}$ \\
\hline & $\mathrm{rF}$ & & & & 0,49 ** & $-0,68$ ** \\
\hline & $\mathrm{ra}$ & & & & $0,53^{* *}$ & $-0,72^{* *}$ \\
\hline \multirow[t]{3}{*}{$\% \mathrm{BNC}$} & $r G$ & & & & & $-0,98$ ** \\
\hline & $\mathrm{rF}$ & & & & & $-0,97$ ** \\
\hline & ra & & & & & $-0,97^{* *}$ \\
\hline
\end{tabular}

$* *=\mathrm{P}<0,01$ e ns $=$ não significativo pelo teste $\mathrm{t}$.

${ }^{1}$ PBNC - prod. de bulbos não comerciais; PBC - prod. de bulbos comerciais; PMBC - peso médio de bulbos comerciais; \% - \% plantas improdutivas; \%BNC - \% bulbos não comerciais; \%BC - \% bulbos comerciais.

Tabela 3. Estimativas dos coeficientes de correlação genotípica $\left(r_{G}\right)$ correlação fenotípica $\left(r_{F}\right)$ e ambiental $\left(r_{a}\right)$ de caracteres de folha, avaliados aos 48 e 69 dias após o transplantio, com caracteres associados à produção, em 62 progênies de meio-irmãos de cebola ValeOuro IPA-11. Juazeiro (BA), UFRPE, 1998.

\begin{tabular}{|c|c|c|c|c|c|c|c|c|c|c|c|}
\hline \multirow{2}{*}{ Caracter } & \multirow{2}{*}{ Correlação } & \multicolumn{2}{|c|}{$\mathrm{CF}^{1}$} & \multicolumn{2}{|c|}{ ANG } & \multicolumn{2}{|c|}{ NTF } & \multicolumn{2}{|c|}{ NFD } & \multicolumn{2}{|c|}{ NINFA } \\
\hline & & 48 & 69 & 48 & 69 & 48 & 69 & 48 & 69 & 48 & 69 \\
\hline \multirow[t]{3}{*}{$\overline{P B N C}$} & $r G$ & $-0,50^{* *}$ & $-0,25^{* *}$ & $-0,49^{* *}$ & $0,67^{* *}$ & $-0,52^{* *}$ & $-0,33^{* *}$ & $-0,70^{\star *}$ & $-0,44^{* *}$ & $-0,81^{* *}$ & $-0,04 n s$ \\
\hline & $\mathrm{rF}$ & $-0,37^{* *}$ & $-0,39^{* *}$ & $-0,09 n s$ & $0,14 \mathrm{~ns}$ & $-0,21 \mathrm{~ns}$ & $-0,22 \mathrm{~ns}$ & $-0,12 n s$ & $-0,35^{\star *}$ & $-0,10 \mathrm{~ns}$ & $-0,13 n s$ \\
\hline & $\mathrm{ra}$ & $-0,36^{* *}$ & $-0,44^{* \text { * }}$ & $0,01 \mathrm{~ns}$ & $-0,10 \mathrm{~ns}$ & $-0,01 \mathrm{~ns}$ & $-0,17 \mathrm{~ns}$ & $0,11 \mathrm{~ns}$ & $-0,35^{\text {* * }}$ & $0,13 \mathrm{~ns}$ & $-0,17 n s$ \\
\hline \multirow[t]{3}{*}{$\overline{\mathrm{PBC}}$} & $r G$ & - & $0,63^{* *}$ & $-0,33^{* *}$ & $-0,38^{* *}$ & $0,55^{* *}$ & $0,74^{* *}$ & $0,53^{* *}$ & $0,74^{* *}$ & $0,41^{* *}$ & $-0,02 n s$ \\
\hline & $\mathrm{rF}$ & $0,52^{* *}$ & $0,47^{* *}$ & $-0,13 n s$ & $-0,29^{* *}$ & $0,35^{* *}$ & $0,40^{* *}$ & $0,17 \mathrm{~ns}$ & $0,49^{* *}$ & $0,13 \mathrm{~ns}$ & $0,13 \mathrm{~ns}$ \\
\hline & $\mathrm{ra}$ & $0,40^{* *}$ & $0,46^{* *}$ & $-0,08 \mathrm{~ns}$ & $0,14 \mathrm{~ns}$ & $0,22 \mathrm{~ns}$ & $0,23^{* *}$ & $0,02 \mathrm{~ns}$ & $0,35^{* *}$ & $0,03 \mathrm{~ns}$ & $0,19 \mathrm{~ns}$ \\
\hline \multirow[t]{3}{*}{ PMBC } & $r G$ & $-0,32^{* *}$ & $-0,69^{* *}$ & $0,58^{* *}$ & $0,34^{* *}$ & $-0,41^{\text {** }}$ & $0,45^{\star *}$ & $0,17 n s$ & $0,40^{* *}$ & $0,47^{* *}$ & $-0,07 n s$ \\
\hline & $\mathrm{rF}$ & $0,13 \mathrm{~ns}$ & $0,17 \mathrm{~ns}$ & $0,04 \mathrm{~ns}$ & $0,07 \mathrm{~ns}$ & $0,05 \mathrm{~ns}$ & $0,24^{* *}$ & $0,14 \mathrm{~ns}$ & $0,28^{* *}$ & $0,21 \mathrm{~ns}$ & $0,15 \mathrm{~ns}$ \\
\hline & $\mathrm{ra}$ & $0,21^{\text {* * }}$ & $0,32^{* *}$ & $-0,07 n s$ & $-0,03 \mathrm{~ns}$ & $0,28^{* *}$ & $0,17 \mathrm{~ns}$ & $0,12 \mathrm{~ns}$ & $0,24^{* *}$ & $0,14 \mathrm{~ns}$ & $0,21 \mathrm{~ns}$ \\
\hline \multirow[t]{3}{*}{$\%$} & $r G$ & $-0,22^{* *}$ & $0,68^{* *}$ & $0,22 n s$ & $0,00 \mathrm{~ns}$ & $-0,59^{* *}$ & $0,37^{* *}$ & $-0,46^{* *}$ & $0,36^{* *}$ & $-0,35^{* *}$ & $0,39^{* *}$ \\
\hline & $\mathrm{rF}$ & $-0,35^{* *}$ & $-0,21 \mathrm{~ns}$ & $0,12 \mathrm{~ns}$ & $-0,07 \mathrm{~ns}$ & $-0,35^{* *}$ & $-0,01 \mathrm{~ns}$ & $-0,22 n s$ & $-0,13 n s$ & $-0,14 n s$ & $0,22 \mathrm{~ns}$ \\
\hline & $\mathrm{ra}$ & $-0,40^{* *}$ & $-0,41^{* *}$ & $0,10 \mathrm{~ns}$ & $-0,10 \mathrm{~ns}$ & $-0,21 \mathrm{~ns}$ & $-0,18 \mathrm{~ns}$ & $-0,13 n s$ & $-0,36^{* *}$ & $-0,08 n s$ & $-0,18 \mathrm{~ns}$ \\
\hline \multirow[t]{3}{*}{$\% \mathrm{BNC}$} & $r G$ & $-0,88^{* *}$ & $-0,69^{* *}$ & $-0,17 n s$ & $0,39^{* *}$ & $-0,74^{* *}$ & $-0,57^{* *}$ & $-0,85^{* *}$ & $-0,65^{* *}$ & $-0,85^{* *}$ & $-0,04 n s$ \\
\hline & $\mathrm{rF}$ & $-0,48^{* *}$ & $-0,49^{* *}$ & $0,01 \mathrm{~ns}$ & $0,25^{* *}$ & $-0,33^{* *}$ & $-0,28^{* *}$ & $-0,15 n s$ & $-0,40^{* *}$ & $-0,13 n s$ & $-0,12 n s$ \\
\hline & $\mathrm{ra}$ & $-0,41^{* *}$ & $-0,47^{* *}$ & $0,06 \mathrm{~ns}$ & $-0,16 \mathrm{~ns}$ & $-0,09 n s$ & $-0,16 \mathrm{~ns}$ & $0,11 \mathrm{~ns}$ & $-0,29^{* *}$ & $0,09 \mathrm{~ns}$ & $-0,14 \mathrm{~ns}$ \\
\hline \multirow[t]{3}{*}{$\% \mathrm{BC}$} & rG & $-0,83^{* *}$ & $0,51^{* *}$ & $0,08 \mathrm{~ns}$ & $-0,36^{* *}$ & $0,79^{* *}$ & $0,43^{* *}$ & $0,77^{* *}$ & $0,51^{* *}$ & $0,71^{* *}$ & $-0,35^{* *}$ \\
\hline & $\mathrm{rF}$ & $0,49^{* *}$ & $0,47^{* *}$ & $-0,04 n s$ & $-0,19 \mathrm{~ns}$ & $0,36^{* *}$ & $0,24^{* *}$ & $0,17 \mathrm{~ns}$ & 0,39 ** & $0,13 \mathrm{~ns}$ & $0,05 \mathrm{~ns}$ \\
\hline & $\mathrm{ra}$ & $0,44^{* *}$ & $0,48^{* *}$ & $-0,07 \mathrm{~ns}$ & $0,16 \mathrm{~ns}$ & $0,14 \mathrm{~ns}$ & $0,16 \mathrm{~ns}$ & $-0,03 n s$ & $0,34^{* *}$ & $-0,04 n s$ & $0,17 \mathrm{~ns}$ \\
\hline
\end{tabular}

** $=\mathrm{P}<0,01$ e $\mathrm{ns}=$ não significativo pelo teste $\mathrm{t}$.

${ }^{1} \mathrm{CF}$ - comprimento de folhas; ANG - ângulo entre as duas folhas centrais completamente desenvolvidas; NTF - número total de folhas; NFD - número de folhas danificadas por tripes; NINFA - número de ninfas nas duas folhas centrais completamente desenvolvidas; PBNC - produção de bulbos não comerciais (kg/parcela); PBC - produção de bulbos comerciais (kg/parcela); PMBC - peso médio de bulbos comerciais; \% I - porcentagem de plantas improdutivas; \% BNC - porcentagem de bulbos não comerciais; \% BC - porcentagem de bulbos comerciais.

adicionais no estágio de pré-bulbo com os maiores danos causados pelos tripes.

Entre caracteres associados à produção, PBC apresentou todas as correla- ções positivas com PMBC e \%BC e negativas com \%BNC (Tabela 2). Correlações genotípicas e fenotípicas positivas e significativas entre $\mathrm{PBC}$ e \%BC em cultivares de cebola também foram obtidas por Dowker et al. (1976), Buso (1978), Candeia et al. (1986) e Carvalho (1996), e para PBC com PMBC por 
Carvalho (1996). Os caracteres PBNC, $\% \mathrm{I}$ e \%BNC, correlacionaram-se de forma positiva entre si e negativas com PBC, PMBC e \%BC. Resultados semelhantes foram relatados por Buso (1978), Candeia et al. (1986) e Carvalho (1996).

Aos 48 DAT (Tabela 3), o caracter NINFA apresentou correlações genotípicas com PBNC $(-0,81 * *)$, \% I ($0,35 * *), \% \mathrm{BNC}\left(-0,85^{* *}\right), \operatorname{PBC}\left(0,41^{* *}\right)$, $\operatorname{PMBC}(0,47 * *)$ e $\% \mathrm{BC}(0,71 * *)$. Estas correlações são contrárias às afirmações de que os tripes causam o aumento do número e da produção de bulbos não comerciais e redução da qualidade e da produção de bulbos comerciais (Sato, 1989). Também foram observadas correlações genotípicas entre NFD e PBC $(0,53 * *)$ e $\% \mathrm{BC}\left(0,77^{* *}\right)$, indicando que mesmo com o aumento do número de folhas danificadas, a produção e porcentagem de bulbos comerciais não foram reduzidos, como seria esperado. Este fato pode estar relacionado à resistência por tolerância das progênies de ValeOuro IPA-11 já que na fase mais suscetível da cultura, quando as plantas iniciam o processo de bulbificação (Gonçalves, 1998), a população de tripes não afetou a produção. Candeia et al. (1998) observaram que esta cultivar apresentou folhagem com alguma resistência aos danos causados por tripes e que, segundo Candeia et al. (1997) e Hamilton et al. (1999), a cv. Roxa IPA-3, um dos genitores da cv. ValeOuro IPA-11, apresentou tolerância ao tripes. Já aos 69 DAT o caracter NINFA correlacionou-se significativamente apenas com $\% \mathrm{I}(0,39 * *) \mathrm{e}$ $\% \mathrm{BC}\left(-0,35^{* *}\right)$ indicando que o maior número de ninfas na planta acarreta o aumento do número de plantas improdutivas e redução do número de bulbos comerciais.

Os pares de caracteres $\mathrm{CF}$ e PMBC, em ambas as avaliações, apresentaram correlações genotípicas $(-0,32 * *$ e $-0,69 * *)$ que indicam que quanto maior o comprimento das folhas, menor o peso médio dos bulbos comerciais. McCollum (1971) observou correlações genotípicas negativas para este par de caracteres. Aos 69 DAT, as correlações genotípicas entre CF e PBC $(0,63 * *)$ e $\mathrm{CF}$ e $\% \mathrm{BC}$ $(0,51 * *)$ foram de acordo com Dowker et al. (1976) os quais obtiveram correlações genotípicas para comprimento de folha e produção de $61 \%$ a $80 \%$.

Aos 69 DAT, as correlações genotípicas de NTF com PBC $(0,74 * *)$, $\operatorname{PMBC}\left(0,45^{* *}\right), \% \mathrm{BC}\left(0,43^{* *}\right), \mathrm{PBNC}$
$(-0,33 * *)$ e $\% \mathrm{BNC}(-0,57 * *)$ indicam que maior produção, porcentagem e peso de bulbos comerciais, objetivos principais do melhoramento, podem ser obtidos quando as plantas apresentam maior número de folhas. Estudos sobre o movimento de assimilados durante a fase de bulbificação demonstraram que as plantas de cebola transferem a maior parte da matéria seca das folhas verdes para o bulbo (Currah e Proctor, 1990), justificando estas correlações.

As correlações entre os caracteres indicam que a produção de bulbos comerciais pode ser aumentada através da seleção para aumento do peso e porcentagem de bulbos comerciais e redução da quantidade de bulbos não comerciais. $\mathrm{O}$ melhoramento para redução da infestação de tripes pode ser mais eficiente através da seleção de plantas com menor NTF e maior ANG. Estes caracteres são de fácil mensuração e menos trabalhosos do que a contagem de ninfas nas folhas. No entanto, esta indicação deve ser tomada com cautela uma vez que o NTF está correlacionado positivamente com a produção, porcentagem e peso de bulbos comerciais. As correlações sugerem que a cv. ValeOuro IPA-11 apresenta resistência a tripes por tolerância.

\section{AGRADECIMENTOS}

Os autores agradecem a FACEPE, ao IPA, ao Eng. Agrônomo Jarbas Florentino de Carvalho, a todos funcionários da Fazenda Rodeador e demais pessoas que contribuíram para a execução do trabalho.

\section{LITERATURA CITADA}

BOIÇA JUNIOR, A.L.; DOMINGUES, E.P. Efeito de dimetoato, monocrotofós e carbaryl no controle de Thrips tabaci Lindeman, 1888, em duas variedades de cebola. Científica, Jaboticabal, v.15, n.1/2, p.67-77, 1987.

BORTOLI, S.A.; CASTELLANE, P.D. TERASAKA, F. Incidência de tripes (Thrips tabaci Lind., 1888) (Thysanoptera-Thripidae) em genótipos de alho (Allium sativum L.). Científica Jaboticabal, v.23, n.2, p.355-366, 1995.

BREWSTER, J.L. Onions and other vegetable alliums. Wallingford: CAB INTERNATIONAL, 1994. 236 p.

BUSO, J.A. Estimativas de parâmetros genéticos de caracteres de planta e bulbo de cebola (Allium cepa L.). Piracicaba: ESALQ, 1978. 132 p. (Tese mestrado)

CANDEIA, J.A.; SILVA, N.; ZANOTTO, M.D. Parâmetros genéticos e correlações em cebola 'Pira Tropical'. Horticultura Brasileira, v.4, n.2, p.17-19. 1986.
CANDEIA, J.A.; MENEZES, D.; MENEZES, J.T; MARANHÃO, E.A.A. FRANCA, J.G.E. Cultivar de cebola amarela ValeOuro IPA-11. In: CONGRESSO BRASILEIRO DE OLERICULTURA, 37., 1997, Manaus. Anais.. Manaus: SOB, 1997. p. 56.

CANDEIA, J.A.; CARVALHO, J.F.; MARANHÃO, E.A.; CAVALCANTI, V.A.L.B.; RODRIGUES, V.J.L.B. Avaliação do nível de resistência de populações de cebola ao tripes e ao "sapeca". In: CONGRESSO BRASILEIRO DE OLERICULTURA, 38., 1998, Petrolina. Anais... Petrolina: SOB, 1998. p. 47. 1 CD-ROM.

CARTWRIGHT B.; McKENZIE, C.L.; MILLER, M.E.; PERKINS-VEAZIE, P.; EDELSON, J.V. Enhancement of purple blotch disease of onion by thrips injury. In: PARKER, B.L.; SKINNER, M.; LEWIS, T. ed. Thrips biology and management. New York: Plenum Press, 1995. p.203-208.

CARVALHO, J.F. Avaliação de progênies de meios irmãos em cebola (Allium cepa L.) para caracteres fitotécnicos. Recife: UFRPE, 1996. 68 p. (Tese mestrado)

COUDRIET, D. L.; KISHABA, A.N.; McCREIGHT, J.D.; BOHN, G.W. Varietal Resistance in onions to thrips. Journal of Economic Entomology, v.72, p.614-615. 1979.

CRUZ, C.D. Programa Genes. Aplicativo computacional em genética e estatística. Viçosa: UFV, 1997. 442 p.

CURRAH, L.; PROCTOR, F.J. Onions in tropical regions. United Kingdom: Natural Resoucers Institute Bulletin n.35, 1990. 232 p.

DOWKER, B.D.; HARDWICK, R.C.; FENNELL, J.F.M.; ANDREWS, D.J. Genotypic and enviromental correlations between leaf growth and bulb size in onion. Annals of Applied Biology, v.82, p.341-348, 1976.

FALCONER, D.S. Introdução a genética quantitativa. Viçosa: UFV, 1981. 279 p.

GONÇALVES, P.A.S. Determinação do nível de dano econômico de tripes em cebola. Horticultura Brasileira, Brasília, v.16, n.2, p.128-131. 1998.

HAMILTON, B.K.; PIKE, L.M.; SPARKS, A.N.; BENDER, D.A.; JONES, R.W.; CANDEIA, J.; FRANÇA, G. Heritability of thrips resistance in 'IPA-3' onion cultivar in South Texas. Euphytica, v.109, p.117-122, 1999.

JONES, H.A.; BAILEY,S .F.; EMSWELLER, S.L. Field studies of Thrips tabaci Lind. with especial reference to resistance in onions. Journal of Economic Entomology, v.28, n.4, p.678-680. 1935. KENDALL, D.M.; CAPINERA, J.L. Susceptibility of onion growth stages to onion thrips (Thysanoptera, Thripidae) damage and mechanical defoliation. Environmental Entomology, v.16, n.4, p.859-863. 1987.

LOGES, V; LEMOS, M.A.; MENEZES, D.; BARROS, R. Efficiency of methods for sampling thrips on onion. In: INTERNATIONAL CONGRESS OF ENTOMOLOGY, 11., 2000, Foz do Iguaçu. Anais ... Foz do Iguaçu: SEB, 2000. p.268.

McCOLLUM, G.D. Heritability of onion bulb shape and size. Estimate from half-sib families. Journal of Heredity, v.62, p.101-104, 1971.

MONTEIRO, R.C. Identificação das amostras de tripes.f-consoli@tamu.edu. Mensagem pessoal. 6 mar. 2001.

SATO, M.E. Avaliação do dano e controle do Thrips tabaci Lindeman, 1888 na cultura da cebola (Allium cepa L.). Piracicaba: ESALQ, 1989. 93 p. (Tese doutorado)

VENCOVSKY, R.; BARRIGA, P. Genética biométrica no fitomelhoramento. Revista Brasileira de Genética, Ribeirão Preto, 1992. 486 p. 